\title{
SOFT X-RAY VARIABILITY AND THE COVERING FRACTION OF ACTIVE GALACTIC NUCLEI
}

\author{
Michael A. Strauss, Kenneth W. Wachter, and Alexei V. Filippenko \\ Department of Astronomy, University of California \\ Berkeley, CA 94720 U.S.A.
}

The variability of soft X-rays $(0.2-2 \mathrm{keV})$ in some low-luminosity type 1 Seyferts may partly be due to an extrinsic mechanism: dense clouds of gas in the broad-line region, opaque to soft X-rays, move across our line of sight to the X-ray emitting portions of the accretion disk (Reichert, Mushotzky, and Holt 1986; Lawrence and Elvis 1982; Halpern 1984). As the clouds move, the covering fraction changes stochastically. Evidence for partial covering of the X-ray source in low-luminosity AGNs has been seen in soft X-ray spectra by Holt et al. (1980) and Reichert et al. (1985).

The X-rays are thought to be emitted from a region of radius approximately five times the Schwarzschild radius of the central black hole (Shakura and Sunyaev 1973). For AGNs that radiate at the Eddington limit with bolometric luminosity $10^{44} \mathrm{erg} \mathrm{s}^{-1}$, the X-ray emitting region then has a radius $d \approx 10^{12} \mathrm{~cm}$. This size is comparable to the expected radii of broad-line clouds, which implies that the soft X-ray light curve can be strongly affected by the Poisson statistics of opaque clouds crossing the line of sight.

Wachter, Strauss, and Filippenko (1988) derive the covariance function of the uncovered area for circular clouds moving with fixed speed across the central source. If the clouds are opaque, this is directly related to the covariance function of the soft X-ray light curve itself. Figure 1 shows this covariance function for $d / r=2$, where $d$ is the radius of the central source and $r$ is the radius of a broad-line cloud. The time unit on the $x$-axis, $r / v$, where $v$ is the speed of the clouds, is the time required for a cloud to move a distance equal to its own radius. The shape of this curve is determined solely from the values of $d / r$ and the covering fraction $f$. In particular, measurements of the covering fraction, the halflife of the covariance function of the X-ray light curve, and its standard deviation divided by the mean value, will allow us to determine $d$ and $r$ independently, as we shall now see.

Figure 2 is a contour plot of the covering fraction $f$ and the ratio of source to cloud radii $d / r$ as a function of the half-life of the covariance function and the inverse of the standard deviation of the fractional area uncovered. This latter quantity is given by the ratio of the mean to the standard deviation of the light curve, divided by $1-f$. As a hypothetical example, suppose that we have a light curve for which measurements of the soft X-ray absorption spectrum give $f=90 \%$. Assume a mean flux of 0.1 and a standard deviation of 0.04 in comparable units. Then the value of the ordinate of Figure 2 is 25 . The leftmost dotted vertical contour corresponds to $f=90 \%$, on which the ordinate of 25 corresponds to a value of $d / r=5.3$. The expected half-life (abscissa) is therefore 1.7 in units of $r / v$. Suppose further that the covariance function calculated from the soft X-ray light curve shows a half-life of 0.5 day. Equating $1.7 \mathrm{r} / v$ to the observed half-life, and taking the velocity of the clouds $v$ to be $3000 \mathrm{~km} \mathrm{~s}^{-1}$, we find $r=0.7 \times 10^{13} \mathrm{~cm}$. Multiplying by $d / r=5.3$ gives $d \approx 4 \times 10^{13} \mathrm{~cm}$. Thus, the use of Figure 2 can lead directly to source and cloud size estimates that can be compared with the results of other, independent methods. 
The electron density $n_{e}$ can be calculated in terms of $d$ and $f$ given the X-ray flux and the ionization parameter, or the flux of the broad component of $\mathrm{H} \beta$.

At the moment, we are suffering from a paucity of data. First, we need to identify AGNs whose soft X-ray variability is clearly extrinsic; this can be determined if the variability in hard X-rays, in which the broad-line clouds are transparent, is negligible by comparison. We emphasize that the process described here must be responsible for some of the variability in any object with a large covering fraction of broad-line clouds, but in practice it is impossible to differentiate intrinsic and extrinsic variability in any source for which the two are of comparable strength.

Second, we want long, uninterrupted, soft X-ray light curves with frequent temporal sampling in order to carry out the proposed analysis. Thus, an AGN should be observed frequently enough that even the shortest variability time scales are adequately sampled, over a period of time sufficiently long that low temporal frequencies are covered. We are currently applying our formalism to create observational strategies that maximize the information content of an X-ray light curve, given constraints of detector sensitivities and pass-bands and length of available observation periods.

We eagerly look forward to the publication of more X-ray light curves and spectra from EXOSAT, and we urge observers to obtain high-quality data for the most appropriate low-luminosity Seyfert 1 nuclei with Ginga and Kvant. Eventually $R O S A T$ and $A X A F$ will provide the largest and most complete set of observations for many AGNs. It will then be possible to derive the parameters discussed above for a substantial number of objects, and to study the systematic properties of the broad-line region.

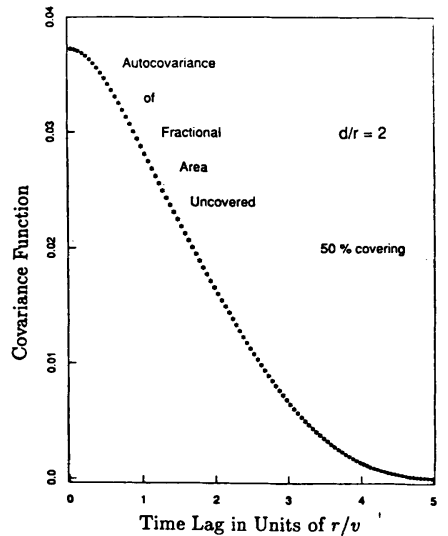

Fig. 1.-A typical example of a covariance function predicted by the model. Here $d / r=2$ and $f=50 \%$.

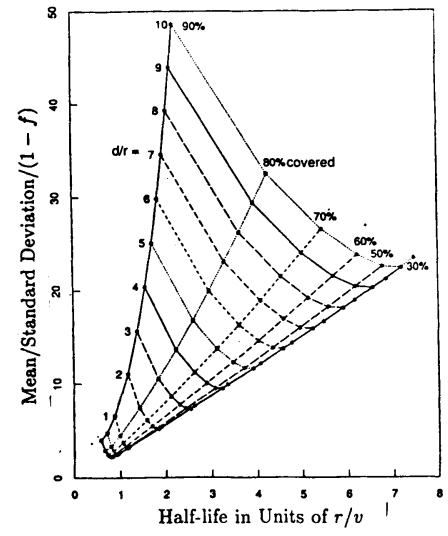

Fig. 2.-Contours of $d / r$ and of $f$, on a plot of amplitude of variability $v s$. covariance function half-life.

Halpern, J. P. 1984, Ap. J., 281, 90.

Holt, S. S. et al. 1980, Ap. J. (Letters), $241, \mathrm{L13.}$

Lawrence, A., and Elvis, M. 1982, Ap. J., 256, 410.

Reichert, G. A., Mushotzky, R. F., and Holt, S. S. 1986, Ap. J., 303, 87.

Reichert, G. A., Mushotzky, R. F., Petre, R., and Holt, S. S. 1985, Ap. J., 296, 69.

Shakura, N. I., and Sunyaev, R. A. 1973, Astr. Ap., 24, 337.

Wachter, K.W., Strauss, M.A., and Filippenko, A.V. 1988, A p. J., 330, 91. 